\title{
Des homes mobiles Zanzibaris : circulations et citadinités de retour d'exil
}

\section{Maïlys Chauvin}

\section{Q OpenEdition}

1 Journals

\section{Édition électronique}

URL : https://journals.openedition.org/eastafrica/484

DOI : 10.4000/eastafrica.484

ISSN : 2790-1076

\section{Éditeur}

IFRA - Institut Français de Recherche en Afrique

\section{Édition imprimée}

Date de publication : 1 juillet 2012

Pagination : 5-20

ISSN : 2071-7245

\section{Référence électronique}

Maïlys Chauvin, «Des homes mobiles Zanzibaris : circulations et citadinités de retour d'exil », Les Cahiers d'Afrique de l'Est / The East African Review [En ligne], 45 | 2012, mis en ligne le 07 mai 2019, consulté le 09 décembre 2021. URL : http://journals.openedition.org/eastafrica/484 ; DOI : https:// doi.org/10.4000/eastafrica.484 


\title{
Des bomes mobiles Zanzibaris : circulations et citadinités de retour d'exil
}

\author{
Mailys Chauvin
}

\begin{abstract}
Résumé - En situation de retour - circulatoire et intermittent - dans la ville d'origine de Zanzibar, les aînés de la diaspora zanzibarie redécouvrent et renégocient leur relation aux lieux quittés quarante à cinquante ans auparavant. Cette citadinité mise en suspens et en cours de reconstruction se fonde sur la superposition de plusieurs villes : celle perdue du passé et de la jeunesse, imaginée ensuite en exil ; celles des nouvelles installations en Europe ou dans le Golfe. La pluralité des expériences citadines et voyageuses révèle un home labile et mobile, cosmopolite et inachevé.
\end{abstract}

\section{Introduction}

Depuis les années 2000, la ville de Zanzibar $^{1}$ est recomposée par le retour des aînés de la diaspora zanzibarie. Fondé sur des circulations spatiales et des intermittences urbaines pluri-annuelles, inscrit dans l'espace transnational fortement connecté des pôles géographiques de la diaspora swahilie mis à jour antérieurement ${ }^{2}$, le retour est ici abordé dans sa dimension symbolique et mémorielle, à partir de la redécouverte et de la renégociation de la relation à la ville d'origine ${ }^{3}$ par les retournés. Cette étude alimente la réflexion plus générale proposée en amont sur la place et le rôle que joue aujourd'hui la ville de Zanzibar dans l'espace de mobilité des populations swahilies, espace recomposé à partir de l'événement insurrectionnel de janvier 1964 qui a poussé une partie de la population de l'archipel à l'émigration. Comment la ville a-t-elle habité les individus entre départ, exil et projet de retour (rêvé/concrétisé) ? Comment en situation de retour se refonde la citadinité de ces habitants en devenir, mise en suspens pendant la moitié d'une vie? Quelle place occupent les lieux de la ville perdue et de la migrance dans ce nouveau voyage? Que nous apprend cette citadinité de la reconquête des productions identitaires et mémorielles exiliques contemporaines ?

\footnotetext{
${ }^{1}$ Zanzibar Town est la capitale de l'archipel du même nom, localisé au large de la côte est-africaine et composant une partie du territoire de la Tanzanie depuis 1964.

${ }^{2}$ Ces résultats ont été présentés au colloque de Gent, (r)Urban Africa: A Multi-disciplinary Approach of the African City, en décembre 2011. Ils sont en cours de publication.

${ }^{3}$ L'analyse présentée ici s'appuie sur plusieurs missions d'enquêtes réalisées à Zanzibar entre 2011 et 2012 et dont la dernière a été financée par l'IFRA (Nairobi, Kenya) ici chaleureusement remercié. Elle est issue de plusieurs dizaines d'entretiens parmi les membres de la diaspora zanzibarie présents à Zanzibar. Un grand merci à eux pour leur sympathique collaboration.
} 
Cette étude s'appuie sur les récits et les subjectivités d'individus recueillis à une période singulière de leur itinéraire de vie et géographique (leur retraite professionnelle en Europe du Nord) et du projet de retour (dans l'île natale Zanzibar) mais aussi celle, historique, de la réconciliation nationale de la société insulaire (maridhiano, kisw.) orientée vers la (re)conquête, par la jeunesse urbaine actuelle, de la souveraineté subtilisée à la génération aînée. Les individus interrogés, pour la plupart, appartenaient à la mouvance nationaliste progressiste de l'aile gauche du ZNP (Zanzibar Nationalist Party) dirigée par Abdulrahman Babu qui devient ensuite le parti UMMA après scission en 1963 (Burgess, 2009 ; 2005). C'est donc ici la ville réconciliée qui constitue le cadre des récentes logiques de retour et de la (re)formulation des discours sur la relation aux lieux d'origine et d'exil. C'est enfin le contexte au sein duquel émerge et est mise en avant une réflexion sur ce qui constitue le home des retournés, notion complexe fortement débattue dans les sciences sociales (Mallett, 2004), omniprésente dans les discours des retournés et assez bien liée au concept d'habiter qui a été jusqu'ici fortement utile à la compréhension des territorialités mobiles et des citadinités polytopiques zanzibaries. Il est donc ici proposé de se familiariser et de s'interroger sur la notion de home dont l'intérêt a été suggéré directement par le contenu des discours des retournés, à travers l'expérience mobilitaire post-révolutionnaire zanzibarie.

\section{La ville perdue des premières expériences citadines}

Zanzibar est la première ville où se fondent l'expérience et la relation citadines des aînés zanzibaris de la diaspora. Les retournés formaient alors la jeunesse urbaine de la fin des années 1950 et des années 1960. Si Zanzibar n'est pas toujours leur ville natale, elle représente malgré tout le lieu où, par naissance ou après installation, se sont forgées leurs identités individuelle et familiale. C'est à partir des lieux et des sociabilités du quartier d'habitation et du voisinage, que se sont élaborées leurs premières appartenances. Leurs pratiques urbaines et leurs appartenances citadines ${ }^{4}$ se déclinent dans l'ensemble de l'espace social et politique de la cité swahilie entre travail, vie étudiante, pratiques sportives (clubs de football fameux), cinéma (trois salles à Stone Town - la vieille ville de Zanzibar Town - entre les quartiers de Malindi et de Vuga) et engagement militant (syndicat portuaire, nationalisme monarchique et progressiste). C'est dans un contexte politique et social urbain singulier que se fonde et évolue leur première citadinité : la lutte pour l'indépendance, la création des premiers partis politiques et des syndicats, l'effervescence intellectuelle, une ville en chantier, créative et pleine d'espérance où chacun semble pouvoir jouer un rôle. Les études sur Zanzibar mettent souvent en relief la violence révolutionnaire qui frappe la ville en 1964 et pendant les années qui suivent, mais négligent souvent de traiter l'activité et l'inventivité politique foisonnantes qui caractérisent la vie urbaine des années 1950 et du début des années 1960. Zanzibar est en effet un centre politique structurant ${ }^{5}$ dans l'espace estafricain fortement connecté aux capitales des pays voisins (Afrique du Sud,

\footnotetext{
${ }^{4}$ Entretiens 2011 et 2012 à Zanzibar. Sur ces thématiques, voir les travaux de Laura Fair (2001), Thomas Burgess (2002) et Brigitte Reinwald (2006).

${ }^{5}$ Entretiens avec d'anciens membres du parti UMMA. Voir également les travaux de James Brennan (2010) et Thomas Burgess $(2001,2005)$.
} 
Mozambique, Ethiopie) et de celles de tous les continents où se sont constitués des mouvements d'émancipation socialistes (La Havane, Le Caire, etc.) et où des progressistes soutiennent ces mouvements africains (Londres).

Survient ensuite un deuxième temps dans l'histoire de la ville qui va, pour cette jeunesse urbaine en effervescence, rompre avec le précédent. Cette rupture se traduit par un conflit intérieur qui ne cessera de l'habiter au cours de son histoire. L'indépendance de décembre 1963 puis l'insurrection de janvier 1964 (qui apparait sous le terme de « révolution » dans les narrations et les écrits officiels) et la création de l'Union avec l'exTanganyika indépendant en avril de la même année, ouvrent la voie à une période de dictature violente et arbitraire. La ville utopique se dilue rapidement. C'est à ce moment qu'une partie de la jeunesse urbaine quitte Zanzibar dans des conditions et sous des contraintes très variables. Prolétaire et intellectuelle, active et pensante, contrainte pour une bonne part à l'émigration, elle n'en a pas moins marqué l'histoire et la sociologie des lieux de la ville et de son identité. Du statut de citadins en prise sur l'avenir de la ville, relégués à celui d'habitants exclus et d'acteurs perdus pour la construction de la cité, ils reviennent aujourd'hui à Zanzibar à la fois étrangers et passeurs de mémoire des lieux. L'« autre voyage », la «nouvelle découverte» (Hove, 2002) que constitue le retour dans le lieu d'origine, signalent moins des retrouvailles avec la ville connue, définitivement perdue, qu'une ré-appropriation et une reconstruction de la citadinité mise en attente des anciens-nouveaux habitants.

Cette évolution politique et sociale zanzibarie duale et contradictoire des années 1960, est à l'origine des sentiments de trahison et de désillusion pour une jeunesse urbaine qui se tourne alors vers les mondes incertains de la dispersion diasporique. Ces conflits intérieurs qui marquent la migrance, sont au cœur de la construction identitaire et de la relation à Zanzibar qui, de ville rêvée à ville trahie puis perdue, est en train aujourd'hui, de ré-émerger en ville apaisée. Les discours des retournés recueillis en situation de retour dans la ville des sociabilités urbaines et des lieux perdus de leur jeunesse, contiennent ce passé citadin qui se combine et se superpose au vécu du présent. Cette incursion du passé dans l'action urbaine ici et maintenant, est dialectique et constitutive de la citadinité, des mémoires et des identités des habitants comme l'exprime Philippe Gervais Lambony (2011) quand il étudie les nostalgies citadines sud-africaines, mais elle prend sans nul doute un contour singulier en situation de retour d'exil.

A.Mf. a 67 ans et vit au Danemark depuis la fin des années 1960. Depuis sa retraite, il passe au moins la moitié de l'année à Zanzibar dans son quartier d'origine, Kiponda. Les premiers temps, il revenait «pour voir», pensant qu'il se lasserait vite. Mais après quelques séjours, il se fait « reprendre » par la ville. Il habite dans la maison familiale qu'il rénove et partage avec les autres membres de sa famille dispersée entre le Golfe et l'Afrique de l'Est. Il construit une maison et plante des arbres sur la parcelle de ses aïeux et désire s'y faire enterrer. Il s'est donc sensiblement ré-investi dans la ville d'origine. Malgré tout, A.Mf. ressent toujours la perte. «I have lost Zanzibar, répète-t-il, I have lost the beauty of Zanzibar, the culture... People were friendly, peaceful, love each other, we didn't have this "you are from here", "you are from there", everybody 
was welcomed, it was not racist». Né au milieu des années 1940, il a 17 ans quand la révolution surgit et 20 ans quand, en 1967, il quitte Zanzibar. Sympathisant de l'aile gauche du ZNP puis membre du parti progressiste UMMA, il se remémore une société urbaine tolérante, éduquée, constructive et politiquement audacieuse et courageuse. Il est nostalgique de la ville "d'avant la révolution » celle de la lutte, de la tolérance et du cosmopolitisme, valeurs auxquelles il s'identifie toujours. Il ne retrouve pas ce passé dont, nous dit l'essayiste et romancier Salman Rushdie (1995), nous sommes tous des émigrés et s'accroche à sa "patrie imaginaire » inventée pour négocier le présent de son retour. A.Mf. évoque à plusieurs reprises les moments et les lieux qui ont structuré les pratiques de son enfance et de sa jeunesse entre la maison familiale de Kiponda, l'école, la plage des baignades matinales sur le front de mer, les meetings politiques, les discussions au baraz̧a à Malindi, les jeux et les discussions avec ses frères et sœurs. Avec son frère A., unique enfant de la fratrie à n'avoir pas quitté le pays, et ses anciens voisins et camarades de classe, ils passent ensemble des heures à bavarder, le matin, pendant la baignade, ou sur les baraza du voisinage, en fin de journée. Ils se remémorent ces moments et ces lieux au fondement d'une ville passée partagée, commune à leur mémoire et au vécu présent de leur citadinité de quartier. Sa ville est au fond celle qu'ensemble ils convoquent par le souvenir et qui s'est aujourd'hui diluée. C'est une ville qui se définit tout autant par un temps - celui de leur jeunesse et des années prérévolutionaires - que par un espace - celui de son quartier et de ses déambulations urbaines.

Cette double temporalité parfois troublante qui habite le retourné en situation de retour dans la ville de ses origines, est bien transcrite par le romancier Dany Laferrière (2009). Son narrateur revenant à Port-au-Prince après des années d'absence tente vainement de l'esquiver: "Je m'étais promis de ne pas regarder la ville avec des yeux du passé. Les images d'hier cherchent sans cesse à se superposer sur celles d'aujourd'hui. Je navigue entre deux temps». Processus familier aux retournés du monde, elle s'apparente pourtant semble-t-il à un passage (obligé) entre deux villes, celle perdue et celle en voie de ré-appropriation, articulé autour de la mobilisation mémorielle et émotionnelle de la ville, par le sujet, du passé et du vécu du retour dans la ville en reconquête et dans laquelle il se projette.

\section{Retour dans les lieux et mémoire du départ}

La situation de retour amène le retourné à renouer avec son quartier, sa famille, ses amis et ses autres relations sociales. C'est par ces canaux que s'oriente la renégociation de sa relation à la ville et qu'il cherche une manière de s'y projeter à nouveau. Mais sa citadinité en reconstruction est aussi influencée par les raisons, les conditions, et le vécu du départ. La "mémoire du départ» expression reprise au romancier zanzibari Abdulrazak Gurnah ${ }^{6}$ qui en avait fait le titre de l'un de ses premiers livres (Memory of Departure, 1987) et qui a lui même connu l'émigration, participe du

\footnotetext{
${ }^{6}$ Abdulrazak Gurnah est écrivain et enseignant-chercheur à l'université de Kent; il est installé depuis la fin des années 1960 en Angleterre.
} 
processus de construction de la ville imaginée pendant l'éloignement. Elle habite d'abord l'espace exilique du retourné et s'invite ensuite dans la réflexion du retourné en situation de retour quand il parcourt à nouveau les lieux laissés derrière lui et qu'il analyse sa façon de renouer avec eux.

La collecte des récits relatifs au départ constitue un corpus des mémoires ordinaires de ce temps si singulier, à la fois déchirant et salvateur, du départ. Les manières de quitter et de parfois véritablement fuir la ville, sont très diverses et révèlent une cité qui n'est plus vivable et qui ne permet plus de se projeter ou pire encore qui représente le danger et la mort. Les récits des retournés témoignent de la diversité des situations et contraintes de départ plus ou moins forcé et urgent mais toujours contraint.

Pour certains interlocuteurs d'origine arabe, indienne, ou comorienne directement menacés par la violence qui surgit le 12 janvier 1964 dans les quartiers de la ville, la mémoire d'un départ soudain et inattendu imposé par les meurtres raciaux qui frappent leurs familles, marque à jamais des individus en exil et les empêche de revenir à Zanzibar. C'est le cas des membres de la famille de F.7, qui restent traumatisés et profondément amers à l'égard des événements qui ont anéantis leur existence zanzibarie et leur projet de vie. Après que des membres de leur entourage familial et amical ont été assassinés, que leur maison de Kiponda abandonnée dans l'urgence pour s'enfuir sur des bateaux de fortune en direction du continent, a été nationalisée par le gouvernement, ils ne peuvent pas envisager un retour. Survivant, F. est l'unique membre de sa famille à avoir pu penser un retour dans sa ville natale à la fin des années 2000. L'acte du retour individuel se réalise dans le cadre d'un projet collectif de solidarité et d'aide à Zanzibar (ONG dédiée à l'assistance médicale) mis en place par des membres de la communauté diasporique indienne d'Angleterre et du Canada. Il n'a plus de famille à Zanzibar, ni de maison. Son retour passe aussi par le projet de récupération de ce bien familial loué par le gouvernement à d'autres personnes jusqu'à aujourd'hui. Lors de la première année de son retour structuré en deux séjours de plusieurs mois à Zanzibar autour de la mise en place du projet d'ONG, son rapport à la ville s'articule entre choix du "pardon » et volonté de réconciliation, souhait de revoir les lieux de la jeunesse traumatisée «pour aller de l'avant» et incertitude quant à la durée de sa présence en ville et à sa capacité à vivre à Zanzibar en-dehors de l'optique du projet. Un an après notre première rencontre, F. a choisi de prolonger l'expérience du retour en s'installant à Zanzibar pour trois ans et en se vouant corps et âme au travail dans l'ONG. C'est la ville où se trouve sa maison, dans le sud de l'Angleterre, et le pays où se trouve ses filles, qui semblent constituer ses points de repère comme une sécurité tandis qu'il avance progressivement dans la reconquête de Zanzibar, ville où il doute cependant qu'il pourra jamais se ré-installer totalement.

En 1972, quelques mois avant l'assassinat du président Abeid Karume, S., originaire de Wete (ville de l'île de Pemba) quitte Zanzibar illégalement de nuit, par bateau, après avoir été emprisonné sept ans sous la dictature, au motif d'avoir tenté de

${ }^{7}$ Discussions informelles entre février 2011 et mars 2012 à Zanzibar. 
s'échapper de l'île (choix qu'il avait fait après que le gouvernement a refusé de lui fournir les papiers dont il avait besoin pour partir étudier à l'étranger). Quelques semaines après être sorti de prison, après des années de mauvais traitements et malgré les menaces que lui fait directement Karume sur une éventuelle nouvelle tentative de fuite, il retente l'aventure risquée d'un départ secret cette fois-ci victorieux. Après une traversée nocturne sur le bateau d'un pêcheur achevée à la nage, il regagne le continent, définitivement libéré de la ville qui était devenue sa prison. Aidé ensuite par des Zanzibaris dans les villes qui structuraient alors l'itinéraire d'exil des Zanzibaris, à savoir Dar es Salaam, Tanga et Mombasa, il obtient un document de voyage temporaire de la Croix Rouge pour quitter le continent en direction du Koweït puis d'Aden et de Mascate où il s'installera, travaillera et formera sa propre famille. De l'expérience de l'injustice, du chaos et de la terreur vécue pendant ses jeunes années à Zanzibar, jointe à celle d'un sauvetage, d'une nouvelle chance et d'une reconstruction, S. affirme avoir retiré une force inébranlable où la peur n'a plus sa place. Cette énergie lui a permis, loin de l'archipel, de penser, de rêver et de construire son retour à Zanzibar, d'où il avait été pourtant chassé et avait subi les pires traitements. Son rapport à la ville se traduit par le souhait profond de renouer avec les siens, de sentir qu'il appartient toujours aux lieux, convaincu que les habitants pourtant longtemps perdus de vue et qui, restés à Zanzibar, le regardent souvent comme un étranger, le ré-adopteront. La violence et l'injustice du départ n'a donc en rien détruit ou anéanti son désir de retour et de retrouvailles avec les habitants et a peut-être même constitué un moteur pour reprendre ce qu'il avait perdu. Ceci rejoint le point de vue de la philosophe indianiste Rada Ivekovic (2012) et de bien d'autres tels Edward Saïd et Salman Rushdie, pour qui tout exil est une figure double : à la fois « perte et gain ", possible création, construction autre. Trois ans après son premier retour, il vit, en 2012, la moitié de l'année à Zanzibar, en deux séjours de trois mois. Sa stratégie de retour en ville qu'il conçoit avant tout comme un retour dans et une réadoption par la communauté des habitants, était fondé sur la construction d'un petit hôtel où il emploie des gens de la ville, et la ré-intégration volontaire des lieux et des moments fondateurs des sociabilités zanzibaries (baraza, mosquées, mariages, etc.). Aujourd'hui il savoure sa victoire : il est parvenu «à appartenir aux lieux $»^{8}$.

\section{De la ville rêvée à la ville réelle : une relation à plusieurs temps}

Pendant l'expérience exilique, différentes étapes marquent le bilan et le repositionnement que réalisent les zanzibaris émigrés vis-à-vis de leur départ, des événements qui ont transformé leur ville, de la distance et de la rupture avec le monde social et culturel dans lesquels ils avaient bâti leurs univers. La relation à leur ville, à leur quartier, aux lieux de la jeunesse et de la violence, évolue et se recompose dans un imaginaire complexe autour de représentations dichotomiques telles que celles du paradis et de l'enfer, du rejet et du manque, du désir et du renoncement. Quand le premier retour survient puis que les suivants se mettent en place, la ville « réelle » se confronte à la ville imaginée pendant l'absence. Généralement le retour et la

\footnotetext{
${ }^{8}$ Entretiens menés en juin et juillet 2011, puis en avril 2012 à Zanzibar Town (île d’Unguja) et à Wete (île de Pemba).
} 
redécouverte de la ville sont vécus entre la pratique des lieux fondamentaux de l'histoire de l'individu et des sociabilités routinières (maison familiale, baraz̧a, marché, front de mer, place de Forodhani, cafés et restaurants internationaux) et le plaisir des relations familiales et amicales retrouvées, notamment avec des individus de la même génération perdus de vue après leur émigration. La ville est en effet le lieu des convergences et des reconnexions des zanzibaris de la diaspora de cette génération. De nombreux retournés s'investissent dans la rénovation ou la construction d'une maison dans la vieille ville ou dans les nouvelles périphéries du nord et du sud de la ville. La difficulté du retour réside dans deux types de découvertes qu'il faut «digérer»: tout d'abord celle de nouvelles valeurs fortement critiquées par les retournés (matérialisme, individualisme, etc.); ensuite, celle de la dégradation, de l'effondrement de la cité comme espace de vie (la ville est perçue comme dégradée, en ruine, sale, inégalitaire, pauvre), et espace de droit (dysfonctionnement des services publics, corruption, désengagement des responsables politiques). À côté de ces frustrations, c'est le plaisir, la joie et souvent l'apaisement que le retour à la ville et la reprise des lieux suscitent chez le retourné, en particulier depuis que la voie de la réconciliation nationale zanzibarie s'est ouverte en 2010 et que des espoirs de changements sont à nouveaux permis.

Nous avons vu que deux temps, deux villes se chevauchent constamment au gré des pratiques et de l'avancée du retour. Ce processus symbolique, mémoriel et temporel qualifié précédemment de "passage», se vit dans le cadre d'une citadinité intermittente et partagée. Le caractère polytopique, alterné et circulatoire de l'habiter des retournés a été mis en évidence antérieurement ${ }^{9}$. La relation à Zanzibar, en reconstruction, semble en effet trouver un équilibre temporaire, dans le partage et l'alternance avec/ dans une (ou deux) autre(s) ville(s) en Europe et/ dans le Golfe.

M., né en 1944, est engagé pendant son adolescence et sa jeunesse auprès des progressistes réunis par le leader Abdulrahman Babu. Accusé en 1972 d'avoir participé à l'organisation de l'assassinat du dirigeant Karume, il est emprisonné pendant 6 ans avec de nombreux autres anciens membres de l'ex-parti UMMA. À sa libération, en 1978, sa famille et l'entourage de celle-ci considérant qu'il pourrait être à nouveau source de problèmes du fait de ses orientations et actions politiques passées, lui demande de quitter Zanzibar ou, selon ses propres termes, le «force à partir». Il part sous la contrainte, avec un sentiment de double punition et d'exclusion de la part de son pays et des siens, vers un pays (Émirats Arabes Unis) inconnu dont le régime politique le repousse. À Dubaï puis Sharja très surveillé par les membres de sa famille qui l'accueillent et contrôlent ses faits et gestes, il se considère "nearly like in another prison ». M. ne s'est jamais senti véritablement chez lui à Dubaï et à Sharja. Il y a travaillé et vécu mais n'y a pas construit de famille, s'y est senti isolé et marginalisé : «(...) I felt isolated and I was discriminated first because I was from Pemba, second because I was comrad $^{10}$ [socialiste] (...) ». Il n'a cessé dès lors d'être nostalgique et de rester du mieux

\footnotetext{
${ }^{9}$ Résultats présentés au colloque de Gent, (r) Urban Africa....

${ }^{10}$ Les Comrads sont une formation politique influente à Zanzibar pendant la lutte pour l'indépendance dans les années 1950 et 1960. Au début de son existence, elle constitue l'aile gauche du parti nationaliste ZNP, un parti très divers dans sa composition et influencé par les grands propriétaires terriens d'origine arabe. Les Comrads
} 
possible relié à Zanzibar par l'intermédiaire des zanzibaris de passage dans le Golfe guettant leurs arrivées, demandant des nouvelles, récupérant les journaux qu'il leur demandait de lui rapporter, envoyant des messages à ses amis proches par leur intermédiaire. Sa ville imaginée est certes la ville des déceptions, de l'injustice et de la violence mais aussi et surtout le lieu de la jeunesse injustement perdu (" I left all my childhood life »), celle de la fraternité, du combat politique avec ses camarades socialistes et de l'UMMA, de l'expérience de la prison où la camaraderie et la solidarité avec deux ou trois de ses co-détenus ont été renforcées. À Dubaï, Zanzibar est omniprésente, quasi-obsédante dans son quotidien : "Sometimes in the night, I woke up, and I was thinking of Zanzibar I was dreaming of Zanzibar (...)». La nostalgie de Zanzibar est renforcée par un sentiment de honte et de culpabilité à l'égard de ses proches et de ses camarades politiques : «I was feeling ashame of myself, why I run from there ? I found myself I had deserted my people. I was forced to do it (...) ». Il revient deux fois à Zanzibar pour quelques semaines: la première fois pour aider sa famille, la seconde pour assister aux funérailles de son camarade Babu. Puis en 2005, il rentre à Zanzibar. La précision de la remémoration du départ de Dubaï et de son excitation confuse à l'arrivée à Zanzibar, l'émotion palpable dans ses mots, témoignent du désir constant qui a caractérisé sa relation à distance avec la ville de Zanzibar. Il répète : "I was quite happy! Quite happy... We were to come together [avec un ami], but there was some problem with the immigration in Dubai. The labor office wanted a paper. (...) I complete the document. I had to stay one day more! oh oh oh, it was horrible... I came to renew my booking again. "Tomorrow, you will leave, 8 o'clock, reporting time!", I was said. It was Kenya Airways. We went 730 am there, checking and everything, quite happy thinking "I am leaving tonight, I am leaving tonight!". About 2 o'clock the plane delayed. 3 o'clock... The plane is not going. It was engine problem. (...) Now what to do? They took us back again, gave us a hotel in Dubai, "Oh it's horrible, difficult". We stayed there for seven hours in the hotel. Then they came back to the hotel. "Alhamdulilai I am going home now !". From Dubaï we went to Nairobi. We had to stay one night, but I was thinking "there's no problem, we are in Africa now !". In the morning we went and at $10 \mathrm{am} I$ was here [à Zanzibar]. Then at this time, I don't know what to do, I can't express my self. I am going up here and here, I want to see this one, I want to see this one, I went to see mister K. [camarade militant et co-détenu] at the

sont également fortement engagés dans la lutte syndicale. Après scission en 1963, quelques temps avant les élections et l'indépendance de l'archipel, ils fondent le parti UMMA, un parti socialiste et anti-racial. L'ASP (Afro-Shirazi Party), mené par Abeid Karume, a alors une bonne audience populaire mais n'a pas de véritable formation politique; il développe une approche raciale anti-arabe. Les Comrads s'opposent tout autant au nationalisme monarchique qu'à une vision raciale du politique et du nationalisme. Ils participent, avec d'autres forces, à l'insurrection et au renversement du régime nouvellement indépendant, à tendance monarchiste, en 1964. En 1972, d'anciens membres de UMMA (le parti est dissout après la révolution) assassinent le président Karume, devenu rapidement après la création de l'Union un dirigeant sanguinaire du régime dit révolutionnaire. Plusieurs dizaines d'entre eux ont été emprisonnés, parfois condamnés à mort puis libérés sous l'effet d'une campagne internationale de soutien conduite par des individus de la diaspora installés en Angleterre, Scandinavie et Allemagne et soutenue par des parlementaires et des intellectuels progressistes d'Europe du nord. Les Comrads étaient donc mal vus des autorités d'Oman et des E.A.U. mais aussi de certains Zanzibaris d'origine arabe venus se réfugier, après les violences raciales de 1964, dans les pays du Golfe, où, encore aujourd'hui, près de cinquante ans après les événements, certains ont la plus grande difficulté à obtenir des visas de tourisme pour rendre visite aux membres de leurs familles émigrées. 
workshop... Alhamdulilai. I don't regret leaving there. I am in peace (...) I found the place backward but I had to face it, I knew before (...)». Pour M., le retour à la ville ("réelle») se traduit comme la tombée de la punition. C'est le soulagement et l'apaisement qui caractérisent le lien renoué avec sa ville, sa maison et son quartier, ses camarades politiques, par delà les difficultés matérielles et relationnelles qu'il rencontre mais qui, pour lui, ne sont que peu de choses après ce qu'il a vécu comme une exclusion de sa ville.

Pour A.Mf., qui adopte vite sa nouvelle ville, Copenhague, y trouve un emploi, fonde une famille avec une danoise, s'intéresse aux valeurs et au mode de vie des danois etc., le premier Zanzibar qu'il se bâtit en migration est celui d'un espace et d'un temps mis de côté. « (...) That time I did not want to talk a lot, to take these things [de la violence à Zanzibar et de Zanzibar] because I was young, I wanted getting a job, doing my life, getting married, but I did not want to think about it too much. I kept it aside. When we were meeting with other zanzibaris we used to talk about it but on my side, I always kept aside ». Puis dans un second temps, en 1984 à l'occasion d'un séjour à Zanzibar, il trouve sa ville fortement changée et abandonnée à son sort. Un sentiment de colère et d'amertume surgit alors. "I started to see how are things, how this place was, the politics, the people ... then these memories came back again. Why is it like this? This place should not be like this, it should be a paradise. Inside my body, I was revolted. I disagreed with every things I was angry, I was bitter». C'est alors une seconde ville qui émerge dans ses représentations. Gâchée, regrettée et perdue, elle l'habite alors d'une nouvelle façon. Ses enfants grandissent et il cherche à leur transmettre son idée et ses souvenirs de Zanzibar. Il les y amène et visite avec eux la parcelle de ses aïeux originaires du Mozambique. Ses filles plantent avec lui un arbre sur cette parcelle où il renouvelle son vœu d'enfance d'y bâtir une maison pour l'habiter pendant ses vieux jours. À partir de sa retraite il y a quelques années, c'est un troisième temps qui s'ouvre. Il ré-organise sa vie et décide de passer plusieurs mois chaque année à Zanzibar. C'est une relation passionnelle à sa ville entre amour ("Zanzibar is my root, I like the island, I love it (...) I love this place Kiponda, I love this house (...) When I am here I forget Denmark »), apaisement ("Now I don't have it anymore, this feeling. I am old, I can't do anything just take care of my life, I want to keep out of politics »), déception et nostalgie persistantes comme nous avons pu le voir au début du texte ("When we meet with others we remember how it was before, in Zanzibar. We criticize the politicians. They only think of themselves. During the old days, there was electricity, there was no problem of water, of malaria the town was clean ... ») qui caractérise alors son retour à Zanzibar.

Pour A.M. qui, envoyé étudier en RDA par le gouvernement révolutionnaire de Zanzibar quelques temps après la révolution, fait le constat qu'un retour est impossible après que le Président Karume et le Comité des $14^{11}$ ont entrepris des actes

\footnotetext{
${ }^{11}$ Le Comité des 14 était composé d'individus issus des jeunesses de l'ASP dont le plus connu était Seif Bakari Ali. Peu éduqués, ils sont à l'origine de ce qui est habituellement nommé la "Révolution de Zanzibar" de janvier 1964. Cellule décisionnelle sous le régime dictatorial et violent du Président Abeid Karume qui émerge
} 
de violence sans précédent (disparitions, assassinats, emprisonnements, torture) c'est d'abord la ville trahie et désillusionnée, invivable et perdue qui l'habite alors. Il ne veut (et ne peut d'ailleurs) pas rentrer. Il effectue une rupture psychologique et rompt avec Zanzibar. Mais assez rapidement il passe à une autre étape, celle de l'indignation. Il attaque ouvertement le régime dans ses cercles de discussion en RDA, puis s'engage, depuis la Suède, dans la campagne internationale de libération des prisonniers politiques conduite par un groupe de zanzibaris d'Europe (Grande Bretagne, RDA, RFA, Danemark, Suède) et dont le centre est à Londres ${ }^{12}$. À travers son engagement s'amorce une possible reconquête de tout ce qui a été perdu la ville. Ce n'est qu'à travers l'action et la lutte qu'il peut ré-envisager la ville de Zanzibar dans son espace intime. Ses amis sont libérés en 1978, et sa relation à la ville évolue à nouveau. Il retourne à Zanzibar quasi annuellement. Pour parvenir à supporter l'ambiance de désorganisation, de division et de haine qui s'est installée dans la capitale tolérante de sa jeunesse et qui l'affecte profondément, il met en place une tactique de retour : ne revenir qu'au moment du Ramadhan, période pendant laquelle les disputes sont mises en suspens. C'est alors la seule solution viable pour « pouvoir faire avec Zanzibar» dit-il. Il achète cependant une parcelle et pense à construire une maison pour ses parents. Leurs maisons avait en effet été démolie par le gouvernement quelque temps après son départ, ses parents qui louent un appartement dans une maison nationalisée à Stone Town, lui avait caché cette tragédie pour l'épargner. Un de ses désirs est donc en quelque sorte de ré-équilibrer cet état de fait. À ce projet très présent dans sa démarche de retour succède un autre : la construction d'une villa pour sa famille sur la côte est, villa qu'il a aujourd'hui transformée en pension avec un ami. La ville qu'il s'imagine passe donc par un chantier de constructions personnelles et familiales. Depuis sa retraite récente, il a passé près de la moitié de son temps à Zanzibar annulant ses billets retour, repoussant les départs, planifiant d'autres projets ... Il se sent depuis peu à nouveau en sécurité à Zanzibar et développe un romantisme lyrique quand il parle des lieux de son enfance, du petit bout de terre qu'il a découvert sur la côte est, enfant, et qui l'a habité pendant les années d'absence pour finalement l'acheter dans les années 1990 : «I think I am reconciliating with Zanzibar now ». La ville de la redécouverte est celle de la réconciliation.

La nouvelle relation à la ville qui émerge en situation de retour peut-être définie comme la remise en marche d'une citadinité mise en attente et singulière, celle de l'habitant à la fois historique et étranger, mobile et pluriel. Elle se fonde sur un double constat partagé par beaucoup de retournés: l'impossibilité de désormais faire sans Zanzibar et, en même temps... d'y vivre en permanence! L'étude précédente ${ }^{13}$ avait montré que les retours à Zanzibar étaient intermittents et construits sur le mode du vaet-vient ou d'une circulation tri-logique entre ville européenne et/ou ville du golfe et Zanzibar. «Living permanently in Zanzibar ? I can't ! » dit le passionnel A.Mf. pourtant fou de sa ville. «I miss my family here, my children, my newspapers ... I can't not live in

\footnotetext{
après l'Union avec le Tanganyika, en avril 1964, ils sont à l'origine de crimes impunis à ce jour (tortures, disparitions, emprisonnements arbitraires, exécutions). La plupart sont aujourd'hui décédés.

${ }^{12}$ La problématique de la constitution d'un espace d'action et de résistance dispersé et mobile des progressistes zanzibaris est traitée dans une étude en cours.

${ }^{13}$ Conférence de Gent, décembre 2010.
} 
Zanzibar permanently, no I can't» admet de son côté A.M. C'est une citadinité intermittente et complémentaire qui constitue le compromis actuel des retournés. Le retour dans la ville natale se double d'une ré-exploration de l'itinéraire de vie, des lieux traversés et adoptés, des relations nouées au cours de la migrance et remet en lumière leurs « patries » alternatives et autres chez-soi pleinement appropriés.

\section{Des villes pour un bome mobile}

En effet, l'analyse du vécu des retournés en situation de retour dans la ville d'origine, montre que cette expérience les pousse à reconsidérer non seulement la relation à celle-ci en situation d'exil et de retour mais aussi celle qu'ils ont entretenue avec la ville du pays (ou des pays) d'accueil, ici Copenhague, Dubaï, Sharja, Stockolm ou encore Berlin. Une troisième, voire une quatrième ville surgit dans cette réflexion tout au long des entretiens.

E. ne cesse d'évoquer Dubaï quand elle parle de sa vie et de son histoire avec Zanzibar. Les deux villes se superposent dans son discours et semblent imbriquées tant dans ses pratiques que dans son système de référence : ses séjours avec sa fille et ses petits-enfants, l'aide qu'elle leur apporte à la maison, le shopping et les habitudes qu'elle a dans les malls, les liens avec les autres zanzibaris et les membres de sa famille ainsi que les mariages à Mascate ${ }^{14}$.

A.Mf. quant à lui parle à plusieurs reprises de Copenhague de sa première épouse et de ses "enfants danois », de son travail, de sa pratique du vélo, de la sympathie qu'il éprouve pour les danois, du droit, de la justice, bref d'un ensemble d'éléments pour lui très importants. «I feel I belong there. The way they live, they are opened, you have the law, people are kind, friendly compare to Norway and Sweden. The more I stayed I understand the way they live, dress, act, eat. If I had to choose between Denmark and Zanzibar? Zanzibar! But between Denmark and Oman, Denmark! It is just the perfect land as long as I know the language. Why the best ? For everything : social, trade unions ... If not Zanzibar, Denmark. I bike there, I love it, for the last 40 years, I bike. Zanzibar is my root, I like the island, I love it. Here I feel really at home. When I am here I forget Denmark unless I talk with my children on the phone. I never miss Denmark here. (..) Home is the place you get peace, free. That's my home. In Copenhagen, I got all the necessities, I got rights, If something bad happens to me, at least I can claim, I have rights.

C'est la ville de Stockholm qui revient sans cesse dans nos entretiens avec A.M. Il voue en effet une véritable passion pour cette ville où il a compris qu'il pouvait véritablement commencer une autre vie. C'est à Stockholm qu'il a senti qu'il était « sauvé », " en sécurité » et où il était politiquement en harmonie avec les orientations du pays. Il parle et décrit avec émotion son quartier de Vaxholm où il explique avoir véritablement fait son nid. Potsdam pour lequel son attachement est moins sentimental

\footnotetext{
${ }^{14}$ Les villes de Dubaï et de Mascate, où vivent deux communautés de Zanzibaris, sont en permanence reliées en avion par les zanzibaris (vol d'une heure) pour des voyages d'affaires, de shopping, et, très souvent, dans le cadre des mariages eux-mêmes très structurants dans leurs pratiques mobilitaires.
} 
revient cependant aussi dans son discours car c'est là qu'il habite désormais avec son épouse et ses enfants. Il parle de leurs sorties et de ses habitudes, de ce qui lui manque quand il est à Zanzibar : les journaux, la presse économique, les sorties au théâtre et aux concerts. Mais il se sent avant tout attaché à la Suède plus qu'à l'Allemagne où il n'a pas le même réseau d'amis et où il se sent plus isolé. Le vécu du retour dans la ville natale pousse le retourné à réfléchir au rôle et au sens que joue chacune des villes dans son histoire et au fait qu'il soit lié aux deux. Il réalise souvent que c'est dans ce binôme que sa vie peut s'articuler.

E. qui a dû quitter Zanzibar après que son mari fut libéré de prison en 1972, part vivre à Dubaï la même année pour financer l'éducation de ses enfants qui eux, s'installent en Inde avec leur père pour suivre leur scolarité. Elle vit plusieurs années à Dubaï seule avant que, après des années d'attente, son mari et ses enfants obtiennent un visa pour la rejoindre. Les enfants y grandissent et y sont éduqués. Lorsque apparaît un conflit à son travail, elle décide de repartir à Zanzibar pour y ouvrir une boutique et faire du commerce " comme son père », commerçant à Pemba avant la révolution, qui avait été mal traité et discriminé en 1964. Ce projet constitue un pied de nez au destin et une ré-appropriation audacieuse de son home originel qui l'avait exclue dès la révolution, ellemême (alors jeune adolescente elle avait été placée quinze jours dans un camp avec sa tante et ses cousins), son père puis son mari et ses amis militants. Depuis qu'elle est réinstallée à Zanzibar, son commerce est florissant. Elle se sent «chez-elle » dans la ville où deux de ses sœurs vivent toujours. Mais Dubaï la rattrape. C'est d'abord là où elle importe ses produits et là aussi que ses petits-enfants grandissent. E. et son mari organisent alors leurs vies (jusqu'à aujourd'hui) entre les deux villes et celles où désormais ils s'approvisionnent et se soignent (en Chine, Thailande et Malaisie). E. a longtemps vécu à Dubaï. Elle connaît bien la ville, y a des relations familiales et amicales. Elle est indignée par son absence de droits en tant qu'étrangère. Sans possibilité d'obtenir un permis de résident ou une carte d'identité donc d'être libre de ses mouvements et de pouvoir avoir accès aux services de santé, E. rejette l'idée de s'y sentir comme chez-elle. Ce sont donc ici les notions de droit, de sécurité et de justice qui servent de repoussoir à la possibilité d'envisager un sentiment d'appartenance à Dubaï. Pour elle, son home c'est Zanzibar. Il est fortement lié aux idées d'origine, d'appartenance (" this is where I belong ») et de famille. Il se définit dans ses mots comme le lieu où elle «se sent bien» au quotidien où «c'est facile» alors que paradoxalement la ville de Zanzibar a été pour elle synonyme d'un vécu très violent, sans compter que la majorité des membres de sa famille vit entre Mascate et Dubaï. Après avoir rejeté catégoriquement de considérer Dubaï ou le Golfe comme son home, elle revient cependant sur son propos et admet que dans les états du Golfe où vit sa famille, la présence de la culture musulmane et arabe, la fait se sentir à l'aise. Elle ne s'y considère pas comme dans un monde étranger. «I love Zanzibar, I am so happy when I am here. But I miss my children and grand-children in Dubaï, so I go there every time, stay three months then come back here for a month because I can not get a visa within a month to go back to Dubaï, they don't allow... When I am in Dubaï I feel fine, I feel at home with my family, I take care of my grand-children, I go for shopping, I buy goods for my shop, but after a while, I miss my Zanzibar and I want to come back home. Here I have my sisters, my brother, I go to the market, it is nice. I like it. The problem is that when I am here, after a while, I want to go back to Dubaï! ». Son home semble donc se dessiner 
dans cette double inscription et appartenance aux lieux et dans la circulation incessante qui relie les membres dispersés de sa famille. Son home n'est-il pas au fond quelque chose qui se rapprocherait des lieux et des mondes de sa famille où qu'elle soit localisée mais de préférence dans un espace cosmopolite marqué par l'islam et l'arabité ?

A.M. considère la Suède et Stockholm comme son home. «I love Sweden ! I consider Sweden like my home, up to now and it will never change ! ». Il s'y sentait enfin en sécurité dit-il après avoir quitté la R.D.A. où le gouvernement révolutionnaire de Zanzibar l'avait envoyé étudier plusieurs années, après être passé par Copenhague où il avait été accueilli par des amis zanzibaris mais où il craignait l'enfermement dans l'entre soi du groupe d'origine. Il y avait trouvé un emploi d'ingénieur dans la capitale et avait commencé a aimé son quartier, Vaxholm, qu'il avait rapidement fait sien. Mais ce qu'il fait qu'il se sentait comme il le dit, " at ease » et en harmonie, c'était le climat politique et la prévalence des idées progressistes qui circulaient alors en Suède quand il ne pouvait ni rester en R.D.A., ni retourner à Zanzibar où il pouvait être inquiété puisqu'il critiquait alors vivement le régime. La Suède faisait parti des pays du Nord où les militants zanzibaris qui avaient lancé la campagne de libération de ses amis politiques, avait trouvé un écho et un soutien. Il a obtenu dans ce home de secours, la nationalité suédoise. Il a pu comme il aime se le remémorer, déchirer son passeport tanzanien. Il refusait en effet cette nationalité imposée après que l'Union entre Zanzibar avec le Tanganyika a signé la fin de la souveraineté de l'archipel. Quelques temps plus tard, il s'installe en Allemagne et construit une famille, conserve sa nationalité suédoise et considère toujours la Suède comme son home de cœur. Potsdam et l'Allemagne représentent aussi un autre bout de home. Revenu à Zanzibar récemment pour quelques semaines à la fin de l'année 2011, il comprend qu'il a besoin de rester vivre à Zanzibar à temps partiel. Il fait renouveler une fois son visa, sa famille allemande mesurant le caractère inévitable et urgent de son souhait. À la question Where is home? A.M. répond sans hésitation : "where I feel at ease! Where I am free, where I am not controlled by anything, where I think I belong to. I was belonging to Sweden. Let me tell you, do you know when I will be home again ?! When I am in Germany with my family! Now there is a split! When I am in Germany, going here [Zanzibar], I say I am going home. It is a split between homes. I identify with both of them, I feel secure now in both of them, I feel at ease now here. But look! Now after three months here I feel I have to go home, I am missing it. I have two homes, I don't prioritize one of them. First, it was only one place: Sweden, Stockholm and very strange, Vaxholm [son quartier], it is a very beautiful place ! I never felt at home in Germany, I missed friends there. But now, from here, I can see I have several homes!». A.M. n'est plus tiraillé entre deux ou trois villes, il fera désormais avec les trois. Son home se situe dans cette multitude.

A.M. «adore» Zanzibar. Il s'y investit beaucoup depuis trois ans : il s'y est remarié, se dédie et investit toutes ses économies dans la parcelle familiale qu'il nettoie, replante et ré-aménage dans le but d'y construire une villa. Ce projet constitue le fil conducteur de son itinéraire : enfant, il avait dit à sa mère qu'il construirait une maison sur cette terre. Adulte, âgé, il tient à respecter sa promesse. C'est sa façon de « s'enraciner » à nouveau dans le lieu historique. Il s'est aussi ré-inséré dans les activités et les sociabilités routinières avec les gens de sa génération, ceux qui sont partis et ceux qui sont restés : baignade à la plage de Mercury, courses au marché de Malindi et 
Darajani, discussions aux baraza de Malindi et Kiponda, sorties nocturnes dans ses bars favoris. Pourtant il ne peut pas se décider à vivre en permanence à Zanzibar. Sa vie et sa famille à Copenhague sont tout aussi importantes. To live here [Zanzibar] for good, it doesn't come up to my mind. I use to say "When I will retire, I will go to Zanzibar" but to live for good, it did not come to my mind. I was thinking I will take a trip one, two, three, four months and get tired of this Zanzibar I will come back to Denmark but intention to come and stay for good, no, I can't go back, economically I can do it, but no, I can't. It is difficult to say why, I think I would got frustrated, you know I grew up in Europe, my mentality, everything .... Still I have the Zanzibar culture but the way I have lived all those years, they can't just go, I have lived more in Copenhagen than here. In Copenhagen I have lived almost 40 years, I arrived there I was 27 years. Here, I got very much upset.You want your things, they don't keep the time punctually, it really frustrates me ... Here every thing is complicated! Really I get bitter ! (...) [Home is] the place you get peace, free. That's my home. I got all the necessities, I got rights, if something bad happens to me, at least I can claim, I have rights ».

Le retour à Zanzibar fait émerger les villes d'attachement et d'identification qui comptent pour le retourné et le conduisent à se réinterroger, confirmer, infirmer, compléter ce qu'il, jusque là, imaginait être ses lieux, ses villes et ses mondes d'identification. En situation de retour "installée» (puisque celle-ci se vit comme un processus qui s'étale sur plusieurs années), il mesure avec plus d'acuité la pluralité et la labilité de ces mondes d'affiliation et de ses appartenances. Non pas qu'il n'en avait pas conscience jusque là ; mais la distance au lieu d'installation lui permet de renouveler son regard.

\section{Conclusion}

L'examen du retour à Zanzibar, circulatoire et intermittent, ici appréhendé sous l'angle du vécu, fait apparaître un processus intéressant: celui de la renégociation de la relation avec la ville d'origine à travers les pratiques présentes par lesquelles s'opère un «retour» mémoriel de la ville perdue. Mais il révèle aussi l'importance dans cette renégociation, d'une autre ville, celle où l'émigrant a pu trouver refuge et bâtir une nouvelle existence, une moitié de vie. La pluralité et la superposition des mondes urbains d'affiliation conduit le retourné à réfléchir à ce qui constitue son bome, terme anglais récurrent de son discours et de sa réflexion pour (re)penser sa relation aux lieux, au passé et au présent.

Tous les retournés montrent un fort attachement à Zanzibar, se définissent et organisent leur avenir par rapport à lui et lui accordent le statut de home mais... sans exclusivité. Au cours de leurs itinéraires géographiques, d'autres lieux, d'autres villes se sont progressivement imposés à eux comme de nouveaux mondes d'appartenance qui, tous ensemble, se complètent et le définissent. C'est ce que le retour met en relief dans leur compréhension de la relation à la ville natale, non pas que cette double, triple, multiple appartenance n'était pas apparue et analysée ailleurs et auparavant, mais en situation de retour, cette réalité surgit avec une conscience toute neuve. Parfois mise de côté certains se surprennent d'ailleurs à découvrir une intimité et un désir nouveaux pour leur ville. 
Le home ni parfaitement localisable, ni exclusivement imaginaire, serait fondé sur la multitude des expériences citadines et voyageuses de l'individu, des mondes sociaux dans lesquels il se serait partiellement ancré et entre lesquels il continue de naviguer. Il se situerait tout autant dans le présent de l'action du retourné que dans la succession des étapes géographiques et personnelles qui jalonnent son itinéraire de vie et continuent de l'habiter. Le home surgit de la pratique mais, fortement imaginé, est aussi là où se trouve l'individu mobile, il se déplace avec lui et se frotte aux conditions présentes de l'expérience, ce qui nous rapproche de la poétique de l'errance défendue par Édouard Glissant (2009) et dont Marion Sauvaire (2011) nous dit qu'« elle n'est pas seulement la traversée d'espace multiples [mais] consiste à assumer des appartenances plurielles qui composent une subjectivité mobile et changeante ». L'étude de la dimension symbolique et mémorielle du retour et de la redécouverte de la ville et des citadinités qui ont fondé le home du retourné, confirme enfin une continuité, celle du caractère cosmopolite, sans frontières et urbain ancien des identités et des territorialités swahilies élargies aux villes et aux mondes des émigrations et des circulations post-révolutionnaires contemporaines.

\section{BIBLIOGRAPHIE}

Barbour J.D., 2007, «Edward Said and the Space of Exil », Literature and Theology, 21(3), pp. 293-301.

Brennan J., 2010, "Radio Cairo and the Decolonization of East Africa, 1953-1964 », in Making a World after Empire, The Bandung Moment and its Political Afterlives, Lee C., Athens, OH : Ohio University Press, pp. 173-195.

Bissell W.C., 2005, «Engaging Colonial Nostalgia », Cultural Anthropology, 20(2), pp. 215248.

Burgess T., 2009, Race, Revolution, and the Struggle for Human Rights in Zanzibar: the Memoirs of Ali Sultan Issa and Seif Sharif Hamad, Athens, OH: Ohio University Press.

-2005, "An Imagined Generation: Umma Youth in Nationalist Zanzibar ", in In Search of a Nation: Histories of Authority and Dissidence in Tanzania, G. Maddox, J. Giblin et

Y.Q. Lawi, Oxford, Dar es Salaam, Athens OH: James Currey, Kapsel Educational Publications, Ohio University Press, pp. 216-249.

-2002, "Cinema, Bell Bottoms, and Miniskirts: Struggles over Youth and Citizenship in Revolutionary Zanzibar", The International Journal of African Historical Studies, 35(2/3), pp.287-313.

-2001, Youth and the Revolution: Mobility and Discipline in Zanzibar, 1950-80, thèse de doctorat, Indiana University.

Di Méo G., 2007, «Identités et territoires : des rapports accentués en milieu urbain ? », Métropoles [En ligne], 1 | 2007, mis en ligne le 15 mai 2007, consulté le 23 mai 2012. URL : http://metropoles.revues.org/80

Fair L., 2001, Pastimes and Politics: Culture, Community and Identity in Post-Abolition Urban Zanzibar, 1890-1945, London, Athens OH: James Currey, Ohio University Press. 
Hove C., 2002, «L'exil disloque la langue : Entretien de Taina Tervonen avec Chenjerai Hove », Paris. http://www.africultures.com/php/index.php?nav=article\&no=2419

Gervais Lambony P., 2011. «Nostalgies citadines et production de l'espace en Afrique du Sud ", in La ville, Wieviorka M. et al., 'Les entretiens d'Auxerre', Auxerre : Sciences Humaines Ed.

Ginsburg R., 1999, " Meditations on Homelessness and Being at Home: In the Form of a Dialogue », in Abbarno, G.J.M. (ed.), The Ethics of Homelessness, vol. 86, Amsterdam : Rodopi.

Glissant E., 2009, Philosophie de la relation. Poésie en étendue, Paris : Gallimard.

Gurnah A., 1996, Admiring Silence, London : Penguin Books.

—1987, Memory of Departure, London : Jonathan Cape Limited.

Hilly M.A., 2006, "Revenir, rester ou circuler?", in Les diasporas dans le monde contemporain, Chivallon C., Berthomière W., Paris, Pessac: Karthala, MSHA, pp. 261-265.

Ivekovic R., 2011, La nouvelle universalité de l'exil. Texte de l'intervention dans le séminaire du Programme 'Exil, création philosophique et politique'. Philosophie et citoyenneté contemporaine, samedi 19 mars 2011, Maison des Associations à Genève. http://revue-exil.com/la-nouvelle-universalite-de-lexil/

Laferrière D., 2009, L'énigme du retour, Paris : Grasset.

Mallet S., 2004, «Understanding Home: a Critical Review of the Literature », Sociological Review, 52(1), pp.62-89.

Reinwald B., 2006, «Tonight at Empire. Cinema and Urbanity in Zanzibar, 1920's to 1960's », Afrique et Histoire, 5(1), pp. 81-109.

Rushdie S., 1993, Patries imaginaires. Essais et critiques, 1981/1991, Paris : Christian Bourgois.

Saïd E., 2008, Réflexions sur l'exil et autres essais, Paris : Actes Sud.

Saleh M., 2008, "Zanzibar outre-mer : l'importance de la diaspora insulaire », in L'autre Zanzibar. Géographie d'une contre-insularité, Bernardie Tahir N. (dir), Paris, Pessac: Karthala, ADES-Dymset, pp. 269-287.

Sarup M., 1994, "Home and Identity», in Traveller's Tales: Narratives of Home and Displacement, Paterson G. et al (ed), London, New York : Routledge, pp. 94-104.

Sauvaire M., 2011, «De l'exil à l'errance, la diversité des sujets migrants. Le cas des romanciers caribéens au Québec », Amerika, 5, pp. 2-10. 